\title{
Blink induced centrotemporal spikes in benign childhood epilepsy with centrotemporal spikes
}

\author{
Takanori Yamagata, Mariko Y Momoi, Masutomo Miyao, Shigeichi Kobayashi
}

\begin{abstract}
A 10 year old girl with benign childhood epilepsy with centrotemporal spikes showed centrotemporal spikes induced by blinking even in a dark room. Spikes could not be induced by photic stimulation, eye closure, eye movement, eye deviation, or passive blinks. There have been no previous reports of spikes induced by blinks in benign childhood epilepsy with centrotemporal spikes.
\end{abstract}

(F Neurol Neurosurg Psychiatry 1997;63:528-530)

Keywords: self induced epilepsy; eyeblink;benign childhood epilepsy with centrotemporal spikes

Epileptiform activity and seizures induced by ocular related phenomena occur among the various stimuli related epilepsies. Most cases involve photosensitive epilepsy in which seizures and epileptiform activity are induced by eye movements or eye closure. ${ }^{1-5}$ Seizures induced by eye closure, blinking, or other eye movements have also been reported in nonphotosensitive patients. ${ }^{4-10}$ The underlying mechanisms may involve flickering effects caused by oscillations of the eyeballs, ${ }^{5}$ sensations from the corneas and conjunctivae associated with eye closure and eye movement, or proprioceptive stimuli from the external ocular muscles. ${ }^{7-9}$ In scotosensitive or fixation off sensitivity the EEG abnormalities last as long as the eyes remain closed. They are due to the elimination of central vision and fixation. ${ }^{11-13}$ There has been one report of a patient who had spikes induced by eyes closed in association with the combined mechanisms of both scotosensitivity and eye movement. ${ }^{14}$ Both photosensitive and non-photosensitive seizures are manifested by generalised discharges on EEG or spikes over the occipital regions.

We report a patient with benign childhood epilepsy with centrotemporal spikes, who exhibited induction of central EEG spikes in response to eye blinking.

\section{Case history}

This 10 year old girl had a history of normal birth and development. There was no family history of epilepsy or other neurological disease. Her first epileptic attack occurred during her sleep when she was 9 years old and consisted of facial spasms around the left side of her mouth and eye deviation accompanied by screaming. After that, left perioral facial spasms were seen on several occasions and were sometimes followed by secondarily generalised tonic convulsions. After treatment with phenobarbitone was initiated, facial spasms and generalised tonic convulsions were not seen. Instead, new attacks consisting of sudden disturbances of consciousness appeared. These attacks involved an interruption of motor activity with repetitive eye blinking, mumbling, and salivation, and lasted from several minutes up to an hour. At times, the patient seemed to fall down slowly or fall asleep after the seizures. The attacks occurred most often during times of physical or emotional stress. Phenytoin was added to the phenobarbitone. However, these attacks continued for several months even when the dosages of phenobarbitone and phenytoin were increased up to $190 \mathrm{mg} /$ day and $230 \mathrm{mg} /$ day respectively. At the age of 10, she was referred to our hospital for evaluation. Physical and neurological examinations were normal. Her intelligence quotient was 77 on WISC-R (verbal 88, physical 69). The results of blood and urine analyses were normal, and serum phenobarbitone and phenytoin concentrations were $34.6 \mu \mathrm{g} / \mathrm{ml}$ and $7.7 \mu \mathrm{g} / \mathrm{ml}$ respectively.

On EEG, the background activity was dominated by a $10-\mathrm{Hz} \alpha$ rhythm with a mixture of slower superimposed waveforms. Slow, diphasic, high voltage spikes were often seen during sleep over the left or right centrotemporal regions and were occasionally bisynchronous (figure, a). The spikes were less frequent during wakefulness than during sleep. These spikes occurred simultaneously with her spontaneous eye blinks (figure, b, c). The latency from the beginning of the blink on an electrooculogram (EOG) to the onset of the spike was approximately $60-70 \mathrm{~ms}$. The time of the eye closure on EOG and the onset of the spike seemed to be simultaneous. The spikes were induced by most single and repetitive blinks, were also induced by eye blinks in a dark room (figure, d), and were confirmed in bipolar recordings (figure, e, f). Voluntary blinks induced spikes 

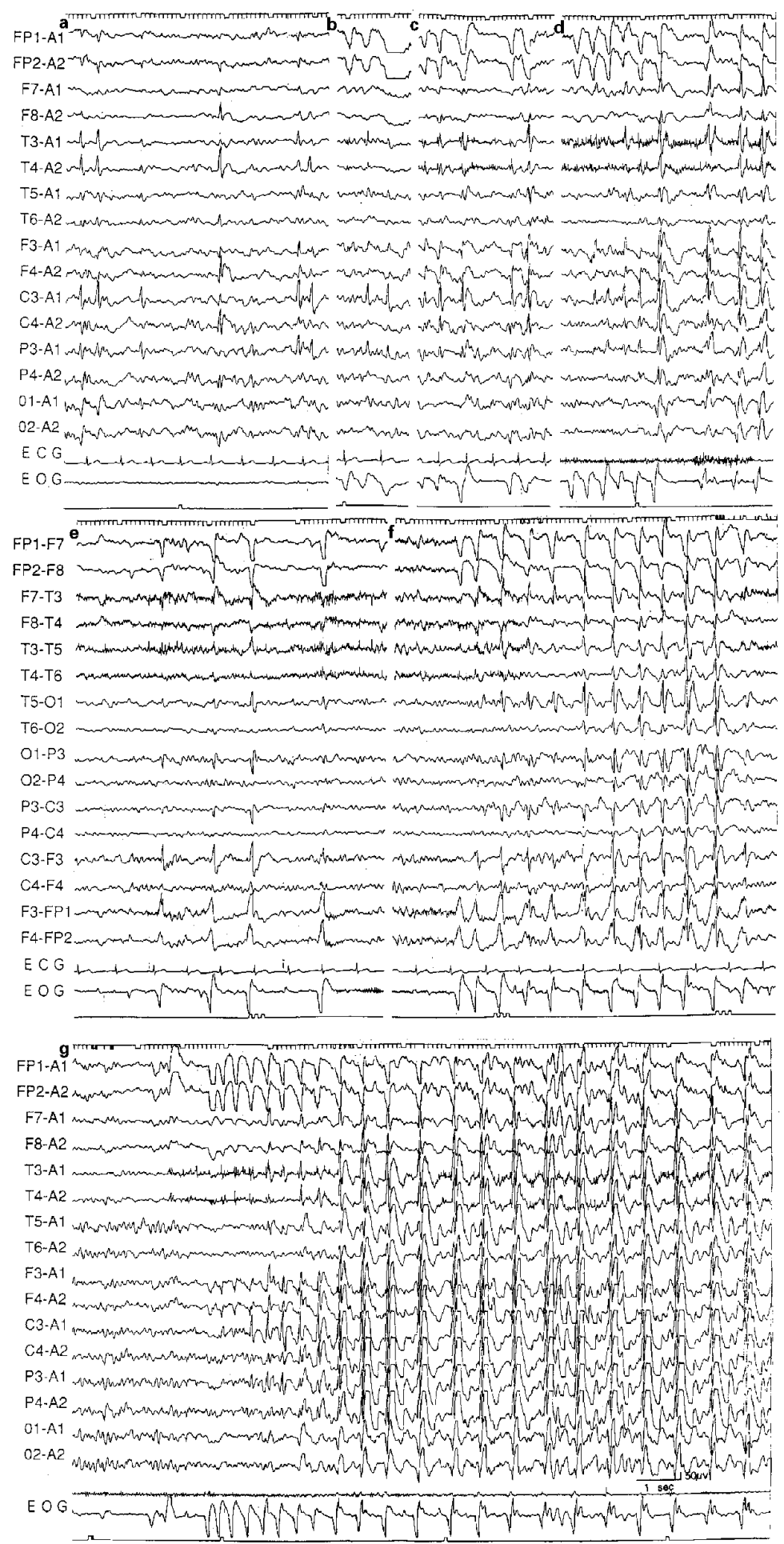

EEG recordings of the patient. (a) EEG recording during light sleep. Frequent centrotemporal, diphasic, high voltage spikes (Rolandic spikes) are present bilaterally. (b and c) single and repetitive spontaneous eye blink induced central spikes or bisynchronous spikes. (d) EEG recording in a dark room. Spikes were also induced in the dark room. (e and f) bipolar recordings of spikes induced by spontaneous blinks (e) and voluntary blinks (f). (g) EEG recording with voluntary blinks. At the beginning of the blinks, left centrotemporal spikes are apparent and occur synchronously with the eye blinks and then gradually generalise with repetitive blinking. Blinks are indicated by EOG.

that appeared in the left central region at the beginning of eye blinking and then gradually generalised (figure, $\mathrm{f}, \mathrm{g}$ ). Sustained eye closure did not induce spikes. The patient's consciousness was mostly preserved during blinks, even when the spikes became generalised. Her con- sciousness became clouded when eyeblinks continued for a long period or when their frequency increased. Photic stimulation with flashing lights, including low frequency stimulation at the rate of her eyeblinks $(1 \mathrm{~Hz}$ and above), and a red lamp, as well as slit or pattern stimulations, did not induce spikes. Eye movements, eye deviation, and passive blinks with palpebral movement by the examiners did not induce the spikes, despite the fact that these passive blinks were induced to obtain voltages and cycles on EOG similar to those of voluntary blinks. Tapping of the leg did not evoke spikes.

The induction of spikes by blinks was impaired after phenobarbitone and phenytoin were gradually reduced, and was not seen after administration of valproic acid.

\section{Discussion}

Our patient was thought to have benign childhood epilepsy with centrotemporal spikes because of her age, the types of seizures, and the presence of centrotemporal diphasic spikes. Of relevance is the fact that these spikes were also induced by voluntary and involuntary eyeblinks, even in a dark room, but could not be induced by various photic stimuli, eye closure, eye movements, or passive blinks. These results ruled out both photosensitive and fixation off sensitive epilepsy. The induction of spikes by sensations from the corneal surface or external eye muscles also seemed unlikely.

There are only two case reports of spike induction by eye blinks only. ${ }^{9}{ }^{10}$ One involved a patient with Lennox-Gastaut syndrome ${ }^{10}$ who showed induction of diffuse spike and wave activity by eye blinks but not by photostimulation, eye closure, or eye deviation. The other was a patient who showed induction of central EEG spikes by eye blinks, similar to our patient. $^{9}$

Most patients with spike induction relating to ocular phenomena showed spikes over the occipital regions or diffuse spike and wave activity. Only a few patients who have exhibited induction of spikes by eye movement have spikes over the frontal region. ${ }^{6}$ Furthermore, only one patient, reported by Nadkarni et al, ${ }^{9}$ showed induction of central spikes by eyeblinks. It was speculated that this spike induction was associated with abnormal, excessive excitatory interconnection between the precentral motor area, from which the voluntary blinks arose, and the epileptogenic zone of the Rolandic cortex. The same mechanism of induction could be postulated in our patient with benign childhood epilepsy with centrotemporal spikes.

In benign childhood epilepsy with extreme somatosensory evoked potentials (ESEPs), central or parietal spikes can be induced by tapping of the feet or other parts of the body. ${ }^{15}$ There has been no report that blinking induced ESEPs. The possibility that eye blinks worked as tapping of the eyelids and induced the ESEPs in our patient cannot be completely ruled out but this was thought to be unlikely because of our failure to induce spikes by passive blinks. 
Self induced epilepsy was also considered in our patient, as she blinked repetitively during periods of stress. Phenobarbitone might have had some effect on the induction of spikes, as the occurrence of blink induced spikes was temporally related to the high dose administration of phenobarbitone and disappeared after gradually reducing the dosage.

Thus, we hypothesise that in the genesis of the eye blink induced spikes, some factors relating to motor potentials, or the eyelid tapping, induced the centrotemporal spikes in this patient with benign childhood epilepsy and that phenobarbitone augmented this effect.

1 Gastaut N, Tassinari CA. Triggering mechanisms in epilepsy. The electroclinical point of view. Epilepsia 1966;7:85-138.

2 Green JB. Self-induced seizures. Clinical and electroencephalographic studies. Arch Neurol 1966;15:579-86.

Watanabe K, Negoro T, Matsumoto A, Inokuma K, Takaesu E, Aso K, Yamamoto N. Self-induced photogenic epilepsy in infants. Arch Neurol 1985;42:406-7.

4 Green JB. Seizures on closing the eyes. Electroencephalographic studies. Neurology 1968;18:391-6.
5 Darby CE, de Korte RA, Binnie CD, Wilkins AJ. The selfinduction of epileptic seizures by eye closure. Epilepsia 1980;21:31-42

6 Shanzer S, April R, Atkin A. Seizures induced by eye deviation. Arch Neurol 1965;13:621-6.

7 Lewis JA. Eye closure as a motor trigger for seizures. Neurology 1972;22:1145-50.

8 Vignaendra V, Thiam Ghee L, Chong Lee L, Siew Tin C. Epileptic discharges triggered by blinking and eye closure. Electroencephalogr Clin Neurophysiol 1976;40:491-8.

9 Nadkarni MA, Postolache V, Gold A, Labar DR. Central mid-temporal spikes triggered by blinking. Electroencephalogr Clin Neurophysiol 1994;90:36-9.

10 Rafal RD, Laxer KD, Janowsky JS. Seizures triggered by blinking in a non-photosensitive epileptic. $\mathcal{f}$ Neurol blinking in a non-photosensitive
Neurosurg Psychiatry 1986;49:445-7.

11 Panayiotopoulos CP. Inhibitory effect of central vision on occipital lobe seizures. Neurology 1981;31:1331-3.

12 Lugaresi E, Cirignotta F, Montagna P. Occipital lobe epilepsy with scotosensitive seizures: the role of central vision. Epilepsia 1984;25:115-20.

13 Panayiotopoulos CP. Fixation off sensitive epilepsies: clinical and EEG characteristics. In: Wolf P, ed. Epileptic seizures and syndromes. London: John Libbey, 1994:55f-56.

14 Barclay CL, Murphy WF, Lee MA, Darwish HZ. Unusual form of seizures induced by eye closure. Epilepsia 1993;34: form of

15 Tassinari CA, De Marco P. Benign partial epilepsy with extreme somato-sensory evoked potentials. In: Roger J, Bureau M, Dravet Ch, Dreifuss FE, Perret A, Wolf P, eds. Epileptic syndromes in infancy, childhood and adolescence. London: John Libbey, 1992:225-9. 